\title{
Evidence Based Practice: Valuable and Successful Examples from Nursing and Midwifery in Rwanda
}

\author{
Marik Moen ${ }^{1 *}$, Innocent Ndateba ${ }^{2}$, Anita Collins $^{2}$, J. D. Iyamuremye ${ }^{3}$ \\ ${ }^{1}$ University of Maryland School of Nursing \\ ${ }^{2}$ University of Rwanda School of Nursing \\ ${ }^{3}$ Ministry of Health, Rwanda
}

\begin{abstract}
While research is needed and necessary, promoting the value of evidence-based practice (EBP), quality improvement (QI) and project evaluation (PE) initiatives could rapidly and economically further the development of nursing and midwifery disciplines globally, perhaps especially in resource constrained settings. We present a review of distinctions between EBP, QI, PE, and research, as well as three examples of successful evidence based practice initiatives: a program evaluation of an EBP course at the University of Rwanda; a study of a teaching strategy aimed at increasing the use of nursing diagnosis to further EBP in nursing; and, the development of an evidence based national model for prevention of Secondary Traumatic Stress in the Health Care worker.
\end{abstract}

Keywords: evidence-based practice, nursing, midwifery, education, quality improvement, research

\section{Introduction}

A brief search for the terms Rwanda and nurse or nursing in 10 renowned health and social sciences databases yielded over 2800 publications since 1985 . The same search for Rwanda and medicine yielded over 5800. A search for Nigeria and nurse or nursing yielded over 9600 publications, while a search for Uganda yielded over 7000, and a search for South African over 42,000. In terms of publications, Rwandan nurses and midwives may appear to be lagging in conducting or generating evidence based practice or research. However, 15 papers and 46 poster presentations at the inaugural Global Innovations in Nursing and Midwifery Education, Research, and Practice Conference with over 300 attendees were a monumental attestation that they are not behind.

While research is needed and necessary, promoting the value of evidence-based practice (EBP), quality improvement (QI) and project evaluation (PE) initiatives could rapidly and economically serve the development of our nursing and midwifery disciplines globally. Proceedings from the conference's Evidence Based Practice Panel provide the rationale for championing EBP and QI nursing and midwifery projects in Rwanda. This paper presents a brief introduction

*Corresponding author: mmoen@son.umaryland.edu of what evidence based practice is and how it has progressed, followed by three successful evidence based practice initiatives.

To define the conversation, we understand research to be a systematic investigation, via formal scientific methods, to develop or contribute to generalizable knowledge (Newhouse, 2007). Evidence based practice is the application of the best available, current, valid and relevant research findings into clinical decision making by integrating one's own clinical expertise and taking into consideration client values and preferences and available resources. EBP involves asking clinical questions, searching research findings, appraising and applying research findings and evaluating outcomes (Eizenberg, 2005; Stokke, Nina, Olsen, Espenhaug, \& Nortvedt, 2014). Quality improvement is a collaborative initiative to improve systems and processes with the intention to improve outcomes usually within one's own institutional setting, often utilizing EBP (Newhouse, 2007). The plan-do-study-act iterative cycle to QI projects was developed by Deming, the father of quality control. Project evaluation is the systematic collection of information about programs (usually applying logic models and evaluation frameworks) to make judgments, improve program effectiveness, and inform 
future development (Centers for Disease Control and Prevention, 2012). Program evaluation can be used in EBP, QI, and research.

Problem. Around the globe, nurses and midwives in practice, and nursing students and faculty in academic settings are often required to do research. A rigorous research study is a very lengthy and resource-intensive endeavor. Further, many nursing investigations are deemed to be research, when they are actually EBP or QI projects. It is important that nurses are able to distinguish between EBP, QI, and research for practical and ethical purposes.

Even an experienced researcher, Dr. Peter Provonost (leader of creating a culture of safety in healthcare), was required, amidst some confusion, to clarify before the U.S. Department of Health and Human Services Office for Human Research Protections, whether an intervention to prevent catheter-associated infections in multiple ICUs was QI or research. The project was indeed QI, applying evidence established by previous research into other settings (Pronovost, et al, 2006).

To determine whether an endeavor is research, EBP, QI, or PE, nurses and team members must ask themselves: what is entailed and what is the goal? Many publications are available to guide this process. Here is a summary of some aspects of distinction.

\section{Intent and Question}

Is the primary intent to test a hypothesis, understand phenomena, to test and evaluate new interventions, process or practice, in order to contribute to generalizable global knowledge? This is research. QI or PE primarily seek to address a clinical or practice problem, or assess, improve, or evaluate existing interventions, practices or procedures (shown to be effective in the literature) within a particular institution or a specific program (Cobb \& Moberg, 2008).

\section{Process, Design, and Data collection}

Is there a research question, a research protocol, a quantitative (with randomization of subjects; comparison of case vs. control) or qualitative design and data collection tools that are validated, reliable, and standardized to reduce bias? This is research, with lengthy processes and strict design and protocols. Al- ternatively, did one identify a problem, gather preliminary data, plan and implement intervention over few weeks, then gather evaluative data via context-specific measurement tool, analyze process, adapt intervention or process, review results, and then adapt again (otherwise known as small test of change)? This is QI, involving rapid-cycle protocol changes where design is flexible (Newhouse, Pettit, Poe \& Rocco, 2006).

\section{Participants/Subjects}

Research subjects are specifically selected to be representative of the total population. One needs enough subjects to have the power to distinguish the difference made by the intervention and consider whether controls are needed. For EBP, QI, or PE a convenience sample of participants is most practical. Likewise, EBP, QI or PE involves almost all beneficiaries, implementers, and stakeholders to facilitate implementation, to make judgments about the program, and to improve or further develop program effectiveness (Cobb \& Moberg, 2008).

\section{Human subjects}

If the objective is to test an established intervention or improve a practice or process in a specific setting, and the evaluation information is used for immediate improvements within that setting, this is not considered human subjects research even if the data is collected in a systematic way. If the institution interacts with or obtains individually identifiable private information for research purposes (see I above) these individuals require human subjects research protections (Cobb \& Moberg, 2008).

\section{Dissemination}

For QI, publication or presentation is allowed but results must not be inferred to a broader population and dissemination usually occurs within institution or within specific QI fora. The intent is to provide effective models, strategies, tools, or benchmarks rather than generalizable knowledge. For research, the intent is to disseminate generalizable information in order to fill a gap in scientific knowledge, and usually published in scientific/research fora (Cobb \& Moberg, 2008). 


\section{Quick Review}

EVIDENCE BASED PRACTICE

To apply the best available research, integrating one's own clinical expertise and client preferences

RESEARCH

To understand a phenomena or test a novel innovation

\section{QUALITY IMPROVEMENT}

To assess an existing practice or procedure shown effective in the literature

PROGRAM EVALUATION

To make judgments, improve, inform current and future programming

Below we present examples of three successful evidence based practice initiatives: a program evaluation of an EBP course at the University of Rwanda, a research study to assess the implementation of a teaching strategy in multiple countries aimed at increasing the use of nursing diagnosis to further EBP in nursing, and, finally how results of research studies led to an evidence based intervention: a national model for prevention of Secondary Traumatic Stress in the Health Care worker.

\section{Evidence-Based Practice: Success Story I}

Despite the positive impact of EBP on quality health care provision and patient care outcomes, nurses rarely use research findings in their practice; rather, they rely on their experience and knowledge, colleagues' expertise and knowledge, and the knowledge learnt at school (Thomson et al, 2001; Estabrooks, Chong, Brigidear, \& Profetto-McGrath, 2005; McCaughan, Thompson, Cullum, Sheldon \& Raynor, 2005; Eizenberg, 2005). Nursing education programs that only emphasize the research process rather than research utilization constitute additional barriers to EBP utilization (Wallis, 2012). In light of these findings, the School of Nursing and Midwifery of the University of Rwanda introduced an EBP course to second year students. Below, we present program evaluation
(PE or QI) results of two surveys of students at the School of Nursing and Midwifery, Rwamagana Campus: the first one involved second year students only (Fig. 1) and the second one involved both second year and third year students (Table 1). Overall, the 42 second year students positively received the EBP course. The vast majority of students answered yes to all of the seven statements evaluating student perceptions about EBP (see Figure 1).

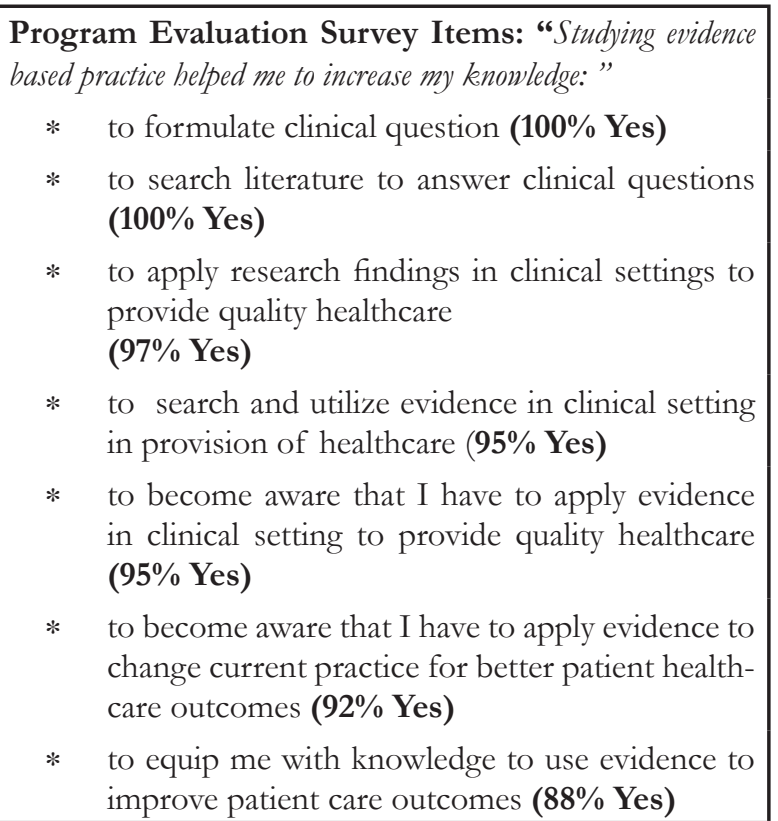

Figure 1. Second year nursing and midwifery students' views on EBP course $(n=42)$

Students in the second survey were asked to respond (yes or no) to statements related to EBP knowledge and skills. Results of the survey presented in Table 1 , show that, in all but one statement, significantly more second year students than third year students answered the questions affirmatively. On average, $79 \%$ of second year students vs. $42 \%$ of third year students answered yes to the statements. 
Table 1. EBP Knowledge of Second $(\mathrm{N}=42)$ and Third Year Students $(\mathrm{N}=35)$

\begin{tabular}{|c|c|c|c|}
\hline Items & $\begin{array}{l}\text { Yes } \% \\
2^{\text {nd }} \text { Year }\end{array}$ & $\begin{array}{l}\text { Yes } \% \\
3^{\text {rd }} \text { Year }\end{array}$ & $\begin{array}{l}\chi^{2} \\
p \text { - value } \\
(\mathrm{df}=1)\end{array}$ \\
\hline I have knowledge about Evidence-Based Practice & 97 & 20 & $<0.001$ \\
\hline I have knowledge about clinical problem or clinical question & 92 & 37 & $<0.001$ \\
\hline I am able to use research findings to answer clinical question & 90 & 48 & $<0.001$ \\
\hline I am able to formulate a clinical question in clinical settings & 95 & 51 & $<0.001$ \\
\hline $\begin{array}{l}\text { I have ability to search literature to answer the clinical problem when } \\
\text { encountered }\end{array}$ & 95 & 82 & 0.078 \\
\hline I am able to use evidence to improve quality health care in clinical setting & 95 & 65 & 0.001 \\
\hline $\begin{array}{l}\text { I am aware that I have obligation to apply evidence in clinical setting to } \\
\text { provide quality care }\end{array}$ & 85 & 42 & $<0.001$ \\
\hline $\begin{array}{l}\text { I am aware that I have obligation to use research findings to change } \\
\text { existing practice for better patient healthcare outcome }\end{array}$ & 90 & 60 & 0.001 \\
\hline $\begin{array}{l}\text { I have adequate skills using MEDLINE or CINAHL databases to search } \\
\text { nursing information in literature }\end{array}$ & 50 & 14 & 0.001 \\
\hline
\end{tabular}

\section{Conclusions}

Many studies indicate a positive relationship between the use of EBP and the quality of care with the ultimate goal to improve patient and healthcare outcomes. Introducing EBP in the nursing and midwifery curricula in Rwanda resulted in positive student perceptions regarding EBP and increased students' knowledge about EBP. Including EBP in nursing and midwifery education may lead to increased application of EBP in healthcare settings.

\section{Evidence Based Practice: Success Story II}

Accuracy in nursing diagnosis is a foundation to evidence based practice according to Levin, Lunney, Miller, Cruz, \& Pimenta (2004) at the Sigma Theta Tau International (STTI) Nursing Congress, Dublin, Ireland. They proposed that EBP cannot be achieved unless the accuracy of nurses' diagnoses is addressed because all other care-related decisions are based on nurses' interpretations of patient-related cues. If nurses' interpretations of patient data are flawed, subsequent decisions regarding outcomes and interventions cannot possibly meet the standards of EBP.

A model was developed to formulate a teaching strategy in India to enhance and increase correct use of nursing diagnoses (see Figure 2). At the heart of the model is work by Levin, Lunney, Miller, Cruz, \& Pimenta (2004) and Sheffer \& Rubensfeld's (2000) seven critical thinking abilities and the ten habits of the mind. The 12-hour teaching strategy was first tested in a study with 41 students (a non-probability convenience sample) of one college of nursing. The findings were presented at the NANDA-I congress at Madrid Spain in 2010. The study was repeated on students and faculty of eight BSc nursing colleges from different states in India with a quasi-experimental design. It was repeated once again, where a control group was used to test the same intervention on 100 nurses from two hospitals. Findings were presented at the NANDA-I $40^{\text {th }}$ anniversary conference in Houston USA in 2012. The results consistently showed a significant improvement in the accuracy of nursing diagnoses and the attitudes towards the nursing diagnosis after the teaching intervention.

The opportunity to implement EBP on a broader scale ensued when the management of Fortis Healthcare Private Limited were interested in using Standardized Nursing Language (SNL) as they wanted to standardize the care across their facilities. Fortis Healthcare has a chain of 73 hospitals, two colleges of nursing, and one school of nursing, with a nursing work force of 17,000 nurses. SNL is based on the concepts of linguistic theories, critical thinking theories and accuracy in nursing diagnosis (ND) theories. The use of SNL helps trace all nursing activities and helped the management engage in key areas of interests: improving patient safety, decreasing cost, improving efficiency and effectiveness. 


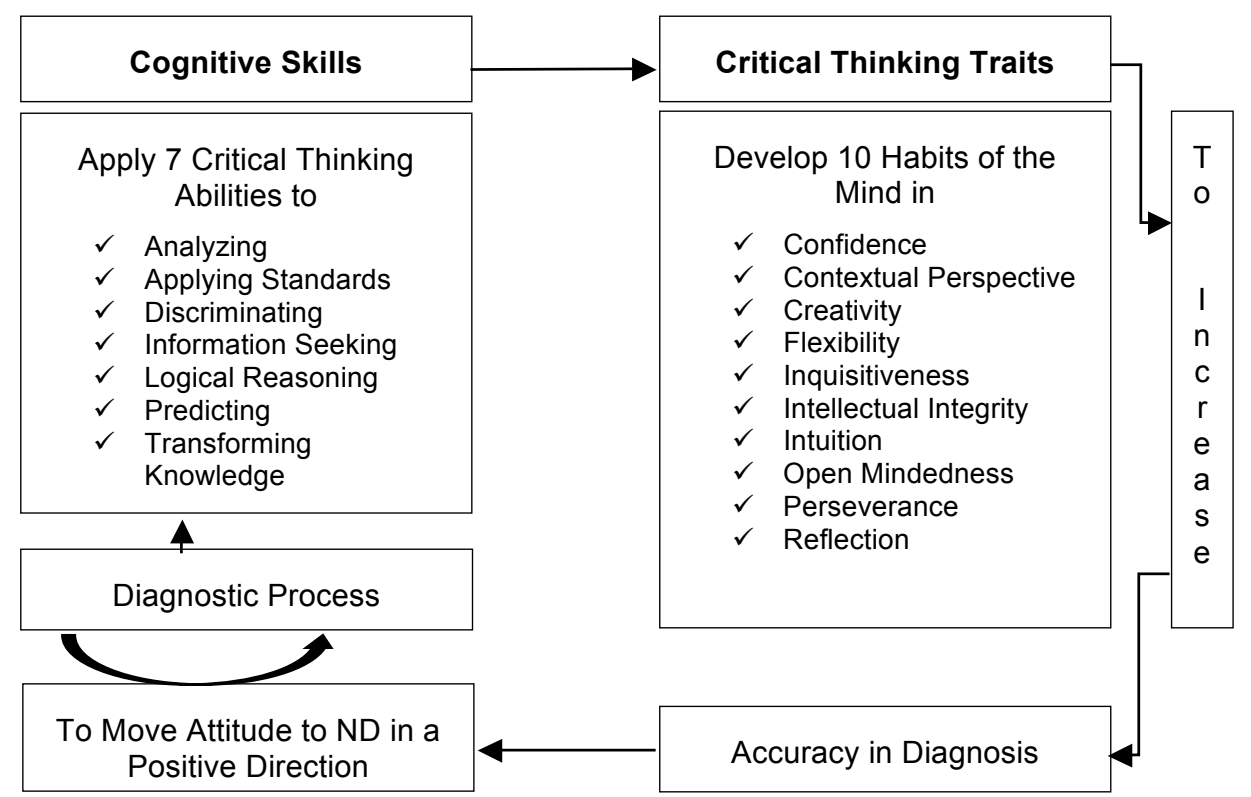

Figure 2. Collins Jagarow Pillai Model for enhancing accuracy and attitude towards nursing diagnoses.

Nursing students had the EBP handbook on ND Nursing Outcomes Classification (NOC) and Nursing Interventions Classification (NIC) while in clinical practice and developed nursing care plans (NCP) from them. Ward nurses began to show interest and requested that these EBP books be made available. Online teaching software helped connect with students at the other schools and colleges as well as nurses in wards to develop accurate NCPs. Gradually, the nurse educators of the hospital and the chief nursing officer began to mandate the documentation of nursing diagnoses by the nurses.

With management's support, a workshop on SNL for institutional nurse leaders was conducted on 27 February 2015 in Rwamagana School of Nursing and Midwifery. The participants interacted, via Webcast, with nurse leaders from countries where SNL is broadly implemented such as Finland, Switzerland, and Spain, to learn first-hand the success and challenges in implementing SNL. There are ongoing negotiations between the University of Rwanda and Elsevier Publishing to procure low cost EBP books for all schools of nursing in Rwanda.

\section{Conclusion}

To implement EBP in education and practice it is vital to engage all stakeholders, most importantly the nurses and students, to build a critical mass with crit- ical thinking abilities and increasingly accurate diagnosing skills. Secondly, it is important to engage the management by highlighting successes so they will be interested in investing time and money for better nursing care. Thirdly, patients must be included, as they are the focus of the attention and are critical for gaining feedback. Finally, the collaboration with other cadre of health workers is integral to successful implementation.

\section{Evidence-Based Practice: Success Story III}

A unique feature of mental health nurses' work involves exposure to clients' descriptions of and reactions to trauma, and these experiences may indirectly cause distress to the mental health worker. This phenomenon has been termed "secondary traumatic stress" (STS) (Iyamuremye, 2010a). The effects of STS can be serious and permanent in mental health workers working with traumatized clients. Two studies were conducted in 2010 to assess STS in the Mental Health workers in Rwanda: A quantitative study of 180 providers of mental health care to trauma survivors using the validated Trauma Attachment Belief Scale (TABS) (Pearlman, 2003), followed by a qualitative study involving the description of mental health workers' experiences of STS (Iyamuremye, 2010a). 


\section{Research to EBP.}

The research provided information about the nature and extent of secondary traumatic stress among mental health workers in Rwanda and supported the development of an intervention model to manage Secondary Traumatic Stress among these workers.
An evidence based intervention model was developed to manage secondary traumatic stress in mental health workers in Rwanda (Fig. 3) and has been implemented nationwide in Rwanda with great success and acceptance as a standard evidenced based practice in mental health (Iyamuremye, 2010b).

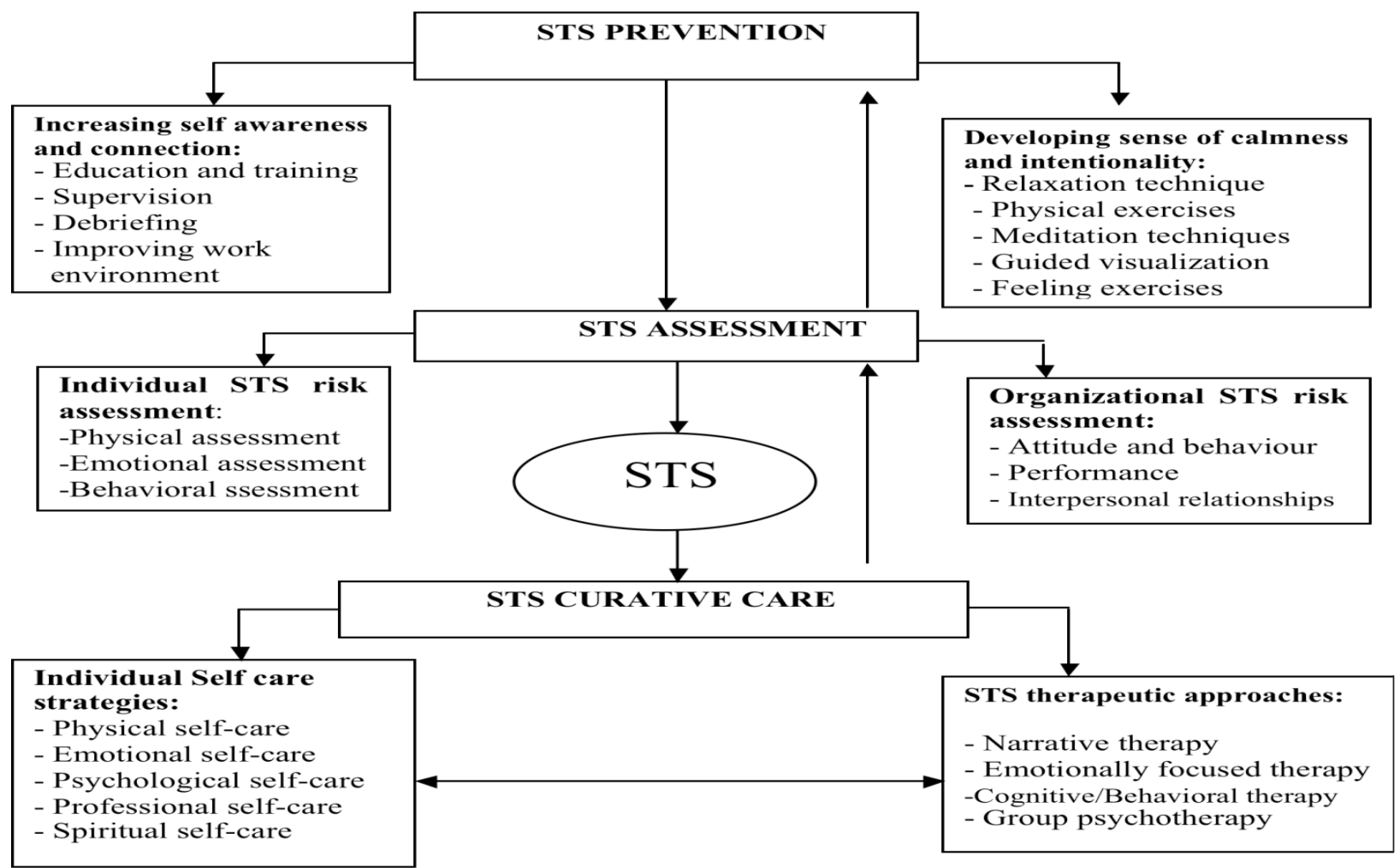

Figure 3. Interventional model to manage STS in mental health workers (Iyamuremye, 2010b)

\section{Conclusion}

The research showed the importance of support being built into the infrastructures of the organizations and mental health services to inform and protect their staff against the possibility of secondary traumatic stress and to offer ongoing support. Improvement in the physical environment is needed to accommodate the mental health workers' break times, and the worker must be encouraged to take breaks during their duty time. Educational programs on secondary traumatic stress need to be developed for the mental health workers, identifying triggers, symptoms and coping strategies. The need to improve mental health service's ability to cope with secondary traumatic stress was evident. Evidence-based recommendations for the mental health workers focused on what the individual can do to recognize, reduce, or prevent secondary traumatic stress effects. Men- tal health professionals need to advocate for their own self-care and to take time to develop personal insights into unresolved issues of his or her trauma.

\section{Recommendations and Conclusions}

As evidenced by these successful EBP-driven projects and the myriad presentations at The Global Innovations in Nursing and Midwifery Education, Research and Practice Conference in Kigali Rwan$\mathrm{da}$, there is the potential for Rwanda to lead globally in EBP via both QI and research. Healthcare and academic institutions must continue to bolster EBP and QI initiatives, ideally with dedicated time and resources for these projects. In the healthcare setting, engaging staff and students in evidence-based reviews of existing (or needed) policies and procedures on each unit, as per Council for Health Service Accreditation of Southern Africa (COHSASA) 
and other accreditation requirements, is a ready opportunity for promoting EBP/QI projects. In academia, nursing and midwifery students at diploma, baccalaureate, and masters levels should be reviewing relevant evidence, and conducting or proposing projects which utilize or test the application of evidence (established via research) in their final projects to improve practices in their own settings. To reduce time and facilitate important work, and to protect healthcare team members (researchers, QI committee members, evaluators) and human subjects, there should be distinctions for review of PE, QI, and research at Institutional Review Boards (IRB) or ethics review boards. Realizing these recommendations and emphasizing the value of EBP and QI would mean that projects on display at this year's conference, and many more that we do not know about, would be broadly championed and disseminated for what they are: successful and laudable accomplishments for nursing and midwifery in Rwanda.

\section{References}

Centers for Disease Control and Prevention. (2012). Introduction to program evaluation for public health programs: $A$ selfstudy guide. Retrieved January 2015 from http://www. cdc.gov/eval/guide/CDCEvalManual.pdf

Cobb, N., \& Moberg, D.P. (2008). Comparison Of The Characteristics of Research, Quality Improvement, And Program Evaluation Activities. University of Wisconsin-Madison Health Sciences IRBs, version date 11/2/08 and Adapted by The Evaluation Center, University of Colorado Denver, 07/09. Retrieved January 2015 from http:// bhpr.hrsa.gov/grants/areahealtheducationcenters/ta/ trainings/materials/ta202qaprogramevaltool.pdf

Collins, A. (2013). Effect of Continuing Nursing Education on Nurses' Attitude toward and Accuracy of Nursing Diagnosis. International Journal of Nursing Knowledge, 24(3), 2047-3095.

Department of Health and Human Services. (2005). Code of Federal Regulations Title 45 Public Welfare Part 46: Protection of Human Subjects, current through April, 2005. Washington, DC: Department of Health and Human Services.

Estabrooks CA, Chong H, Brigidear K, Profetto-McGrath J. (2005). Profiling Canadian nurses' preferred knowledge sources for clinical practice. Canadian Journal of Nursing Research, 37, 118-40.

Eizenberg, M. M. (2005). Implementation of evidence-based nursing practice: Nurses' personal and professional factors. Journal of Advanced Nursing, 67(1), $33-42$.
Iyamuremye, J. D. (2010a). Exploring Secondary Traumatic Stress In Nurses. Nurse Working In Mental Health Services In Rwanda. Berlin, Germany: LAP LAMBERT Academic Publishing.

Iyamuremye, J. D. (2010b). The Intervention Model to Manage Secondary Traumatic Stress In Rwanda Mental Health Workers. Berlin, Germany: LAP LAMBERT Academic Publishing.

Levin, R. F., Lunney, M., Miller, B.K., Cruz, D.M., \& Pimenta, C.M. (2004). Evidence Based Nursing (EBN) \& Diagnostic Accuracy. Presentation at the Sigma Theta Tau Nursing Congress, Dublin Ireland.

McCaughan, D., Thompson, C.A., Cullum, N.A., Sheldon, T.A., \& Raynor, P. (2005). Nurse practitioner and practice nurses' use of research information in clinical decision making: Qualitative findings from a national study. Family Practice, 22, 490-497.

Newhouse, R. (2007). Diffusing confusion between evidence based practice, quality improvement and research. Journal of Nursing Administration, 37(10), 432-435.

Newhouse, R.P., Pettit, J.C., Poe S., \& Rocco, L. (2006). The slippery slope: Differentiating between quality improvement and research. The Journal of Nursing Administration, 36(4), 211-219.

Pearlman, L.A. (2003). Trauma and Attachment Belief Scale. Los Angeles, CA: Western Psychological Services.

Pronovost, P., Needham, D., Berenholtz, S., Sinopoli, D., Chu, H., Cosgrove, S., Sexton, B., Hyzy, R., Welsh, R., \& Roth, G. (2006). An intervention to decrease catheter-related bloodstream infections in the ICU. New England Journal of Medicine, 355 (26), 2725-32, 2801-4.

Scheffer, B. K., \& Rubenfeld, M. G. (2000). A consensus statement on critical thinking. Journal of Nursing Education, 39(8), 352-359.

Stokke,K., Nina, R., Olsen, N. R., Espehaug, B., \& Nortvedt, M. W.(2014). Evidence based practice beliefs and implementation among nurses: A cross-sectional study. BMC Nursing, 13(8), 1-10.

Thompson, C., McCaughan, D., Cullum, N., Sheldon,T. A., Mulhall, A. \& Thompson, D. R. (2001). The accessibility of research-based knowledge for nurses in United Kingdom acute care settings. Journal of Advanced Nursing, 36, 11-22.

Wallis, L. (2012). Barriers to implementing evidence-based practice remain high for U.S. nurses: Getting past "We've always done it this way" is crucial. American Journal of Nursing, 112 (12), 15. 